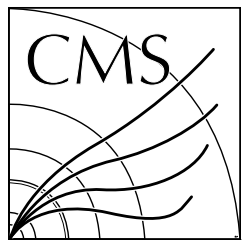

The Compact Muon Solenoid Experiment

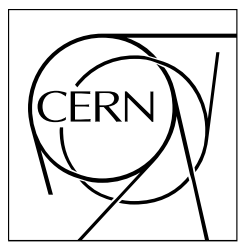

April 2, 2007

\title{
High Voltage System for the CMS Electromagnetic Calorimeter
}

A. Bartoloni, L.M. Barone, F. Cavallari, I. Dafinei, D. del Re, M. Diemoz, S. Guerra, E. Longo, P. Meridiani, G. Organtini, A. Palma, R. Paramatti, F. Pellegrino, S. Rahatlou, C. Rovelli, F. Santanastasio

Dipartimento di Fisica, Università "La Sapienza” and INFN, Sezione di Roma, Roma, Italy.

\begin{abstract}
The CMS electromagnetic calorimeter (ECAL) is made of about 75000 lead tungstate crystals. The 61200 crystals of the barrel part are read by avalanche photodiodes (APD) with internal amplification of the signal. Since the gain strongly depends on the bias voltage, the APDs require a very stable power supply system. To preserve the high energy resolution of the calorimeter, a stability of the bias voltage of the order of $10^{-4}$ is required over several months, a typical interval between absolute calibrations of the full read-out chain with physics events. This paper describes the High Voltage power supply system developed for CMS ECAL and its performances as measured in laboratory tests and during test-beam operations of several modules of the calorimeter.
\end{abstract}




\section{Introduction}

The Electromagnetic Calorimeter of the Compact Muon Solenoid (CMS) [1] detector at the Large Hadron Collider at CERN is a hermetic homogeneous calorimeter [2] made of 61200 lead tungstate $\left(\mathrm{PbWO}_{4}\right)$ crystals mounted in the barrel part, closed by 7324 crystals in each of the two end-caps. The calorimeter is designed to perform precision measurements aiming to reach $0.5 \%$ energy resolution at high energy. The relatively low light yield of $\mathrm{PbWO}_{4}$ (about 100 photons/MeV at $18^{\circ} \mathrm{C}$ ) and the presence of a very intense magnetic field inside CMS (4 Tesla) have led to the choice of APDs (Avalanche Photo-Diodes) as photon sensors in the Barrel and VPTs (Vacuum Phototriodes) in the Endcaps.

The CMS APDs [3], produced by Hamamatsu Photonics, are silicon photodiodes with internal amplification. Two APDs are attached to each $\mathrm{PbWO}_{4}$ crystal. They operate at gain $\mathrm{M}=50$. Since the gain has a quite high dependence on the bias voltage $\left(\alpha_{V}=1 / \mathrm{M} \cdot \mathrm{dM} / \mathrm{dV} \simeq 3.1 \% / \mathrm{V}\right.$ at gain 50$)$, the APDs require a very stable power supply system, as gain fluctuations directly contribute to the constant term of the energy resolution ${ }^{1)}$. A custom high voltage (HV) power supply system has been designed for the CMS ECAL in collaboration with the CAEN Company ${ }^{2)}$. This paper describes this HV system and its performances as measured in laboratory tests and during test beam operations of the calorimeter.

\section{System requirements}

The APDs are Hamamatsu type S8148 reverse structure (i.e., with the bulk n-type silicon behind the p-n junction in order to reduce the nuclear counter effect) specially developed for the CMS ECAL. Each APD has an active area of $5 \times 5 \mathrm{~mm}^{2}$ and two of them are organized in a support (capsule) and glued to the back of each crystal. In total there will be 122400 APDs. The operation at gain 50 requires a voltage between 340 and $430 \mathrm{~V}$. The main electrical properties of the APDs at $\mathrm{M}=50$ and $\mathrm{T}=18^{\circ} \mathrm{C}$ are reported in Table 1 .

Table 1: Electrical properties of the APDs at $\mathrm{M}=50$ and $\mathrm{T}=18^{\circ} \mathrm{C}$.

\begin{tabular}{|l|r|}
\hline Operating voltage & $340-430 \mathrm{~V}$ \\
\hline Difference between breakdown voltage and operating voltage & $45 \pm 5 \mathrm{~V}$ \\
\hline Voltage sensitivity of the gain $(1 / \mathrm{M} \cdot \mathrm{dM} / \mathrm{dV})$ & $3.1 \pm 0.1 \% / \mathrm{V}$ \\
\hline Temperature sensitivity of the gain $(1 / \mathrm{M} \cdot \mathrm{dM} / \mathrm{dT})$ & $-2.4 \pm 0.2 \% /{ }^{\circ} \mathrm{C}$ \\
\hline Maximum dark current (start of experiment) & $<50 \mathrm{nA}$ \\
\hline Typical dark current (start of experiment) & $3 \mathrm{nA}$ \\
\hline Typical dark current after $2 \times 10^{13} \mathrm{n} / \mathrm{cm}^{2}($ end of exp.) & $5 \mu \mathrm{A}$ \\
\hline
\end{tabular}

The stability of the bias voltage seen by the APDs, directly affects the constant term of the ECAL energy resolution through the voltage gain sensitivity. The design goal for the constant term is $0.5 \%$, including all the contributions (e.g. channel intercalibration, light collection uniformity of the crystals, energy leakage). The contribution to the constant term, due to the HV stability, should not exceed $0.2 \%$. To satisfy this request, the voltage stability has to be of the order of $60-65 \mathrm{mV}$ (at $\mathrm{M}=50$ with $1 / \mathrm{M} \cdot \mathrm{dM} / \mathrm{dV}=3.1 \pm 0.1 \% / \mathrm{V}$ ). This requirement is applied to the combination of electrical system characteristics: noise, ripple, voltage regulation and absolute precision, for short and long term periods. This lead to a tolerance of $\pm 20 \mathrm{mV}$ on each contribution.

To avoid the power supply radiation damage during LHC operation, the HV system will be located in the CMS service cavern, around $120 \mathrm{~m}$ away from the detector. Logistic requirements (such as cabling or space in the patch panel) force to feed many APD pairs with the same HV source. The number of APD capsules sharing the same HV channel was then fixed to 50 (100 APDs). The APDs are sorted according to their operating voltage into bins $5 \mathrm{~V}$ wide; then the APDs of the same bin are paired into capsules, so that each pair has a mean gain of 50. As shown in Figure 1, each capsule receives the bias voltage through an RC filter network and two protection resistors $\left(R_{p}=68 \mathrm{k} \Omega\right)$ to limit the bias current to $3 \mathrm{~mA}$ in case of APD breakdown. The HV channels must be floating; they use sense wire to correct for variations in the voltage drop on the leads. The architecture is shown in Figure 2.

\footnotetext{
1) The standard parametrisation of energy resolution is: $(\sigma(E) / E)^{2}=(S / \sqrt{E})^{2}+(N / E)^{2}+C^{2}$ where $\mathrm{S}$ is the stochastic term, $\mathrm{N}$ the noise and $\mathrm{C}$ the constant term

2) CAEN, Viareggio, Italy, www.caen.it
} 


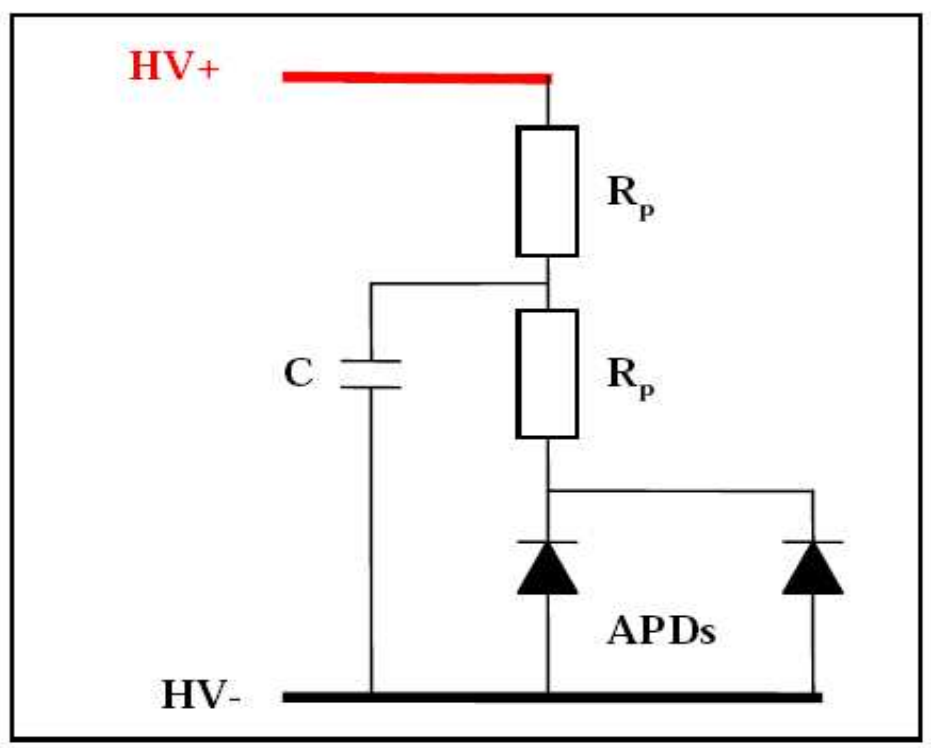

Figure 1: CMS-ECAL APDs loading electrical scheme. $R_{p}=68 \mathrm{k} \Omega$ are the protection resistors. $C=10 \mathrm{nF}$ is a ceramic capacitor. The APD equivalent scheme is shown in pag. 156 of [2].

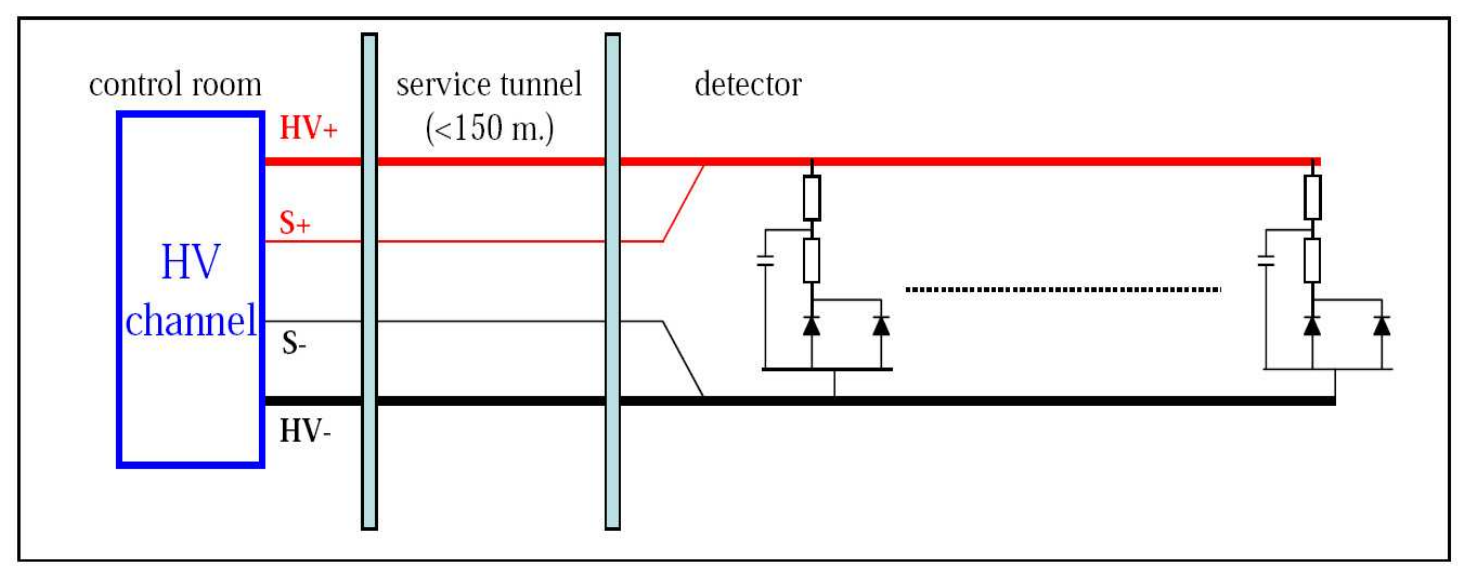

Figure 2: CMS-ECAL APDs HV distribution architecture 


\section{Structure of the HV system}

The system developed by CAEN for CMS ECAL is based on a standard control crate (SY1527) hosting 8 boards A1520E expressly designed for this application (HVBOARD). The SY1527 contains a micro-computer capable to communicate with the board controller via an internal bus and different interfaces are available to integrate the SY1527 on the ECAL detector control system (DCS, see Sect. 8). The board design is based on a modular concept so that each HV channel is implemented as a separate module and up to 9 channels can be hosted on a single HVBOARD, thus permitting a major flexibility in case of channel failure. In total, the system is composed of 18 crates SY1527 and 144 boards A1520E. Each channel is designed to give a bias voltage to 50 capsules from 0 to $500 \mathrm{~V}$ with maximum current of $15 \mathrm{~mA}$; this value allows an $\mathrm{HV}$ channel to work even in the case of short circuit of some of its APDs (four, considering the maximal current over the protection resistors $R_{p}$ ).

The ECAL barrel consist of $2 \times 18$ mechanical units, called supermodules. All the connections are done through a patch panel at the outer end of the supermodule. Each supermodule of the calorimeter contains 1700 crystals, requiring $34 \mathrm{HV}$ channels, taken from 4 boards (one spare channel out of two boards is left). The connection from the board (sitting in the service cavern) to the patch panel is made by custom 9-channel multiwire cables, produced on ECAL specifications by the firm Pansystem ${ }^{3)}$. On the other side of the patch panel, 4 bundles of 8 or 9 multiwire cables go to the so called motherboards, hosting the bias distribution circuits shown in Figure 1 and the connections to the Very Front End electronics (VFE). The motherboards are placed immediately outside the aluminium grid supporting the crystals. Each motherboard feeds through kapton cables a matrix of $5 \times 5$ crystals, corresponding to a trigger tower. Two motherboards are then connected in parallel to the same HV channel.

\section{Laboratory tests on prototypes}

Laboratory tests were systematically performed on prototypes and final modules with different loads: resistors surging the maximum current from all the HV channels of a board at the same time; a programmable electronic load CHROMA 6312 Mainframe equipped with a E63105 module (1A/10A - 125V/500V - 300W) from the firm CHROMA $^{4)}$, capable to generate different load waveforms and to operate in constant current, constant resistance and constant voltage modes; an APD box containing 50 APDs with their bias circuit installed inside a thermostatic chamber. During the measurements different instruments were used and in particular an HP3458A digital multimeter with $8 \frac{1}{2}$ digits resolution that, due to an internal calibration capability, allows the necessary accuracy in temperature and time for long term stability measurements (at 300 Volts presents $1.9 \mathrm{mV}$ over 90 days accuracy and $55 \mu \mathrm{V} /{ }^{\circ} \mathrm{C}$ thermal stability) and a RM-103 Ripple \& Noise Meter from Keisoku-Giken corporation ${ }^{5)}$ that automatically make the measurement of the different components of noise: AC ripple (low frequency: $50 \mathrm{~Hz}$ $2 \mathrm{kHz}$ ), DC ripple (high frequency: $2 \mathrm{kHz}-100 \mathrm{MHz}$ ), noise.

The HV Channel was developed with the following architecture; a DC-DC converter generates the High Voltage (about $500 \mathrm{~V}$ ) from an intermediate voltage of $48 \mathrm{~V}$ and then a linear regulation used to obtain the programmable output voltage with the high stability and ultra low noise figures required by the specification. A micro-controller integrated circuit, embedded on each HVBOARD, controls and monitors, as specified, all the channels hosted on the board and provides a link for communication to a PC.

Test results done on prototypes showed compliance with the specifications regarding long term stability (up to 1 month), load regulation, repeatability, ripple and noise. Variations out of the specification were observed due to temperature changes in the laboratory.

Thermal stability was also verified in the expected environmental temperature range of the power supply rack location $\left(15^{\circ} \mathrm{C}\right.$ to $\left.40^{\circ} \mathrm{C}\right)$ using a thermostatic chamber. Prototype results, in excess of the desired $20 \mathrm{mVpp}$, led to a solution based on more stable components (1ppm accuracy). Temperature drift compensation setting was implemented to improve the boards performance. Temperature drift compensation is possible due to the presence on the crate of temperature probes that can be used to monitor the environment temperature and consequently to regulate the channels output voltage setting. Although this feature is not activated at the moment (see section 6), it can be used anytime.

\footnotetext{
3) Pansystem s.r.l., Rome, Italy, www.pansystem-italia.it

4) CHROMA, www.chromaate.com

5) Keisoku-Giken, www.keisoku.co.jp
} 


\section{Calibration procedures}

An external automatic setup for HV channels calibration completes the system in order to guarantee:

- output voltage precision

- current measurement precision

The automatic setup allows the calibration of one HV board at a time and consists of a Calibration Crate (CAEN SY 3527) equipped with a CAEN T1520 P board connected via serial links to an AGILENT $6 \frac{1}{2}$ digits digital multimeter; the multimeter is read out by the SY3527.

The T1520 P board, expressly designed for this application, contains resistors to simulate different loads during the calibration activities and communicates with the board under calibration through a CAEN Local Net link (CAENET). The calibration setup is sketched in Figure 3.

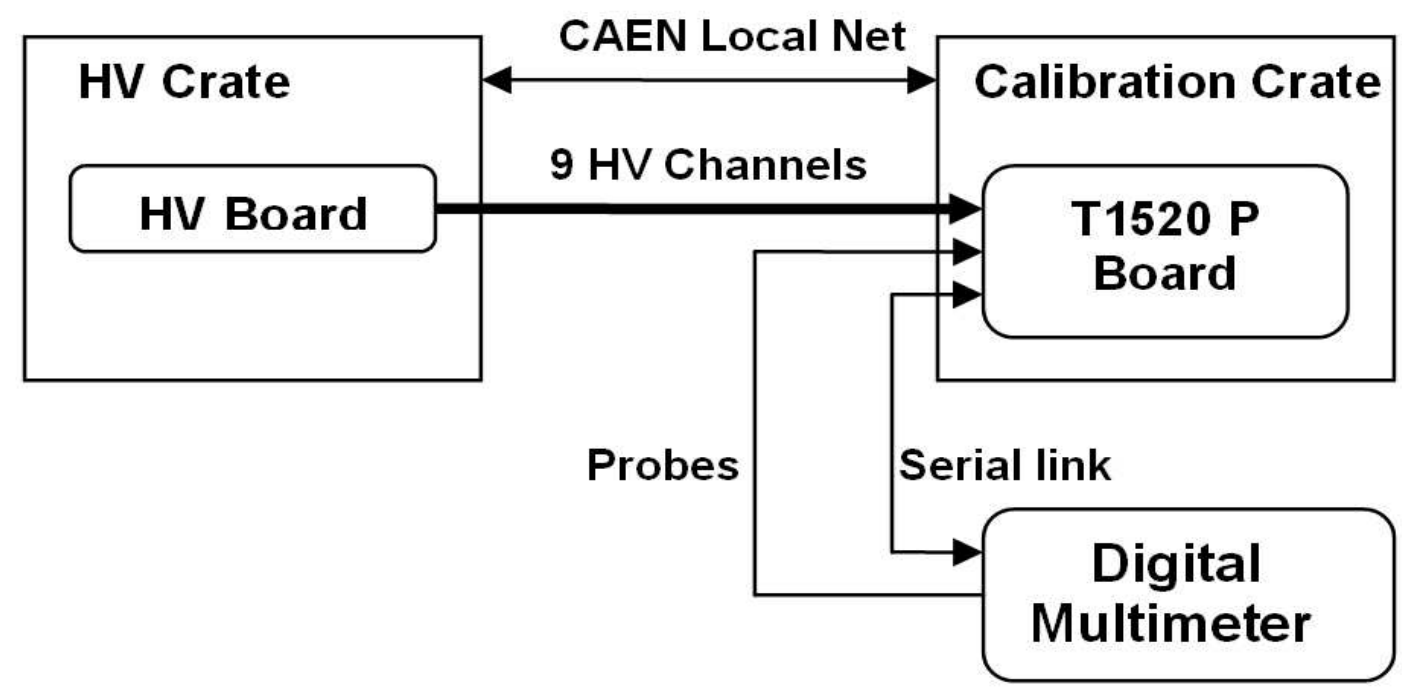

Figure 3: High Voltage channel calibration setup.

In the voltage calibration of each channel, 10 different values of voltage are set in the range $0-500 \mathrm{~V}$ and the corresponding output values are measured with the multimeter and fitted with a polynomial of $3^{r d}$ degree to estimate the parameters for channel calibration; this parameters are then used in the output voltage setting. The same methods are used for current measurements precision. In this case 10 different values of the current in the range $10-100 \mu \mathrm{A}$ are produced by the T1520 $\mathrm{P}$ board, measured with the multimeter and used to calibrate the channel. This kind of calibration will be repeated periodically during the shutdown phases of the experiment.

\section{Stability test}

An acceptance test on the long term stability is performed on each A1520E board on reception. The test foresees setting a voltage $\left(\mathrm{V}_{\text {set }}\right)$ of 380 Volt with a load of $33 \mathrm{k} \Omega$; the corresponding output voltages of the 9 channels are measured every 10 minutes, for around 30 days. Two examples of the output, measured as a function of time, are shown in Figure 4: on the left a stable channel, on the right the channel presents a drift during the test. In both cases the day-night variations are clearly visible. The temperature in the laboratory is measured by 5 sensors located around the crate. Day-night variation and voltage-temperature correlation are shown in Figure 5; in the right plot, a temperature sensitivity of the voltage well inside the specifications is observable (slope of the fit: $\left.d V / d T \sim 3 \mathrm{mV} /{ }^{\circ} \mathrm{C}\right)$.

With the APD working at gain 50, a variation of the bias voltage of $65 \mathrm{mV}$ corresponds to $0.2 \%$ variation in the gain, going directly in the constant term of the ECAL energy resolution. In almost all the channels which present a variation greater than $65 \mathrm{mV}$, the voltage measurements show a clear drift upwards or downwards and not a 

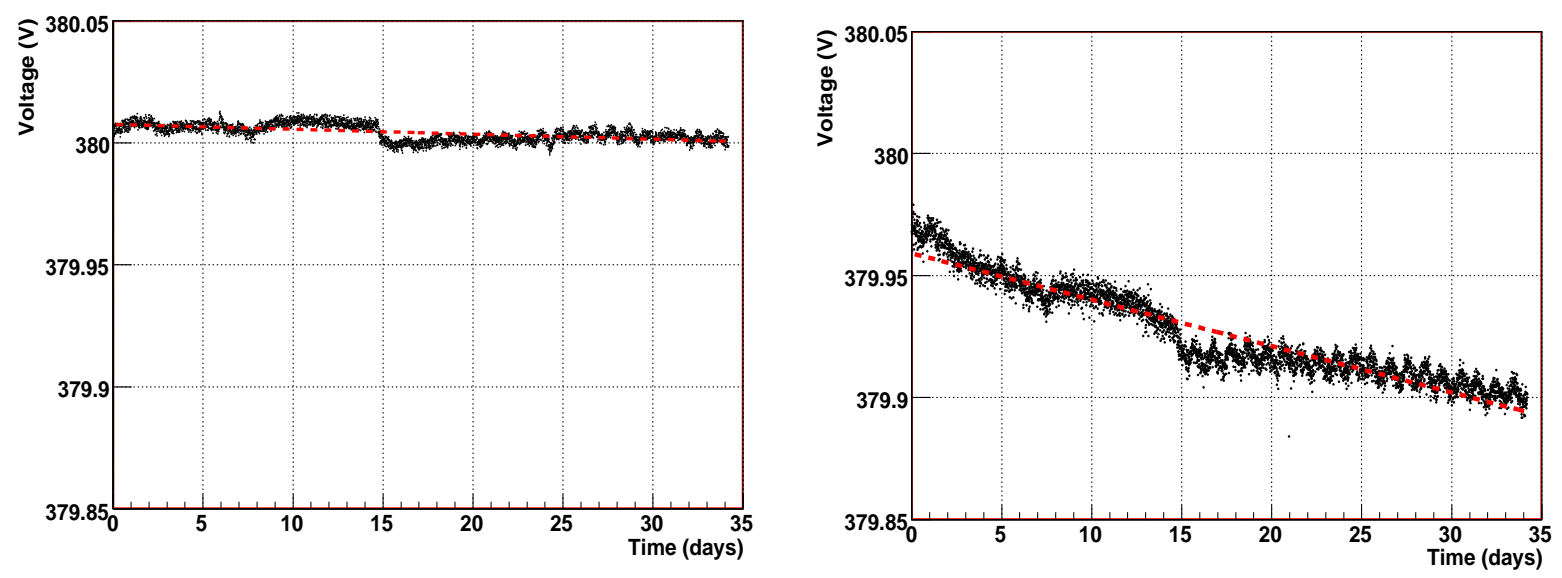

Figure 4: Output voltage as a function of time; on the left a stable channel, on the right a channel outside the specifications.
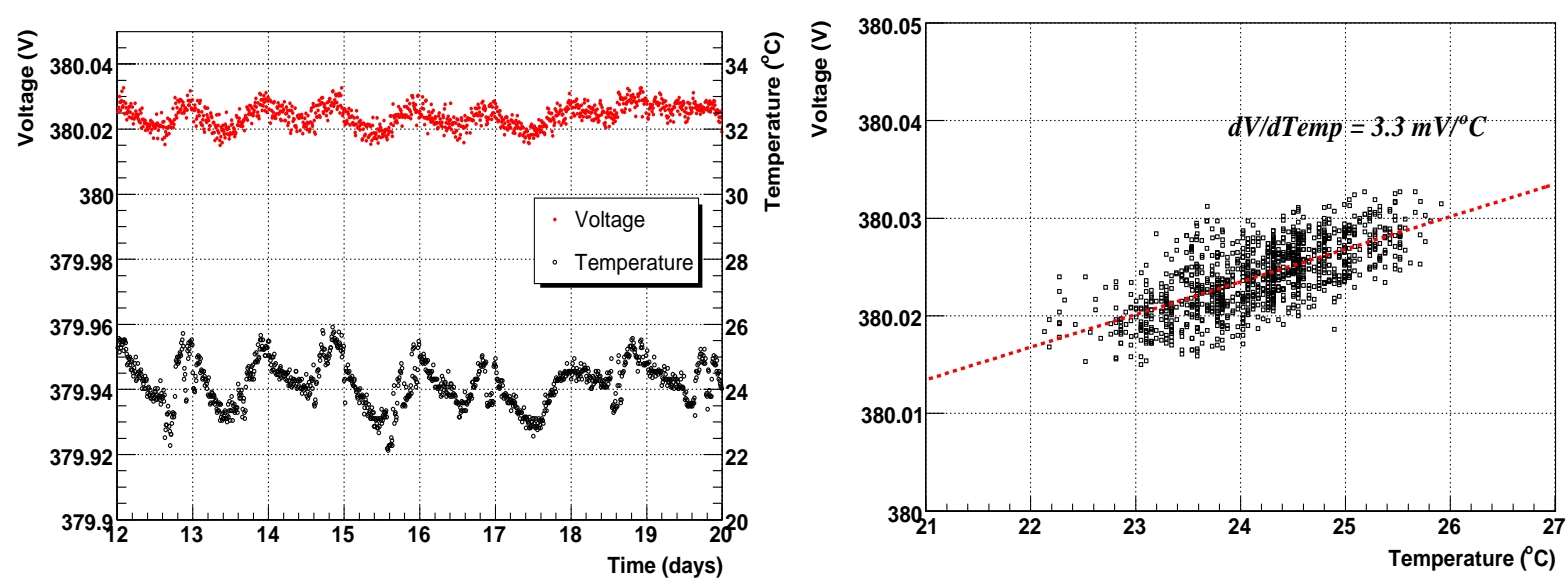

Figure 5: Average of the 5 temperature measurements obtained by the temperature sensors located in the laboratory and voltage measurements taken in the same time interval. On the right voltage values vs temperatures are shown; a linear fit is superimposed.

gaussian-like behaviour; this is due to a drifting component in the channel. A channel is accepted if, after the fit of $\mathrm{V}(\mathrm{t})$ with a first order polynomial, the following conditions are satisfied:

- $\Delta V_{\max }=\max (|V(t)-380.0|)<65 \mathrm{mV}$

- fit slope $<2 \mathrm{mV} /$ day

The distribution of $\Delta V_{\max }$ is shown in Figure 6 for all the channels; the temperature variation effect is not taken into account.

All the data, collected during the stability test, are inserted in a mysql database called Redacle [4]; this database has been originally developed by the Rome Group for the ECAL construction activities performed in the INFN/ENEA Regional Center. A different version of Redacle, adapted for the High Voltage system, is used to trace the crate locations, to mantain the correspondence between boards and crates, and to collect the stability test data.

\section{Performance of the High Voltage system in CMS Test Beam}

During the summer of 2004, the first full ECAL supermodule was tested for a few weeks with high energy electrons at the H4 beam line at CERN. During 2005 and 2006, the beam setup was used to take cosmic ray data with several supermodules in order to calibrate the crystals. In 2006, new beam tests were made with several supermodules. All these tests were done with the final ECAL electronics, a precise cooling system, the laser monitoring and the final HV system installed with full length cables (120 metres). 


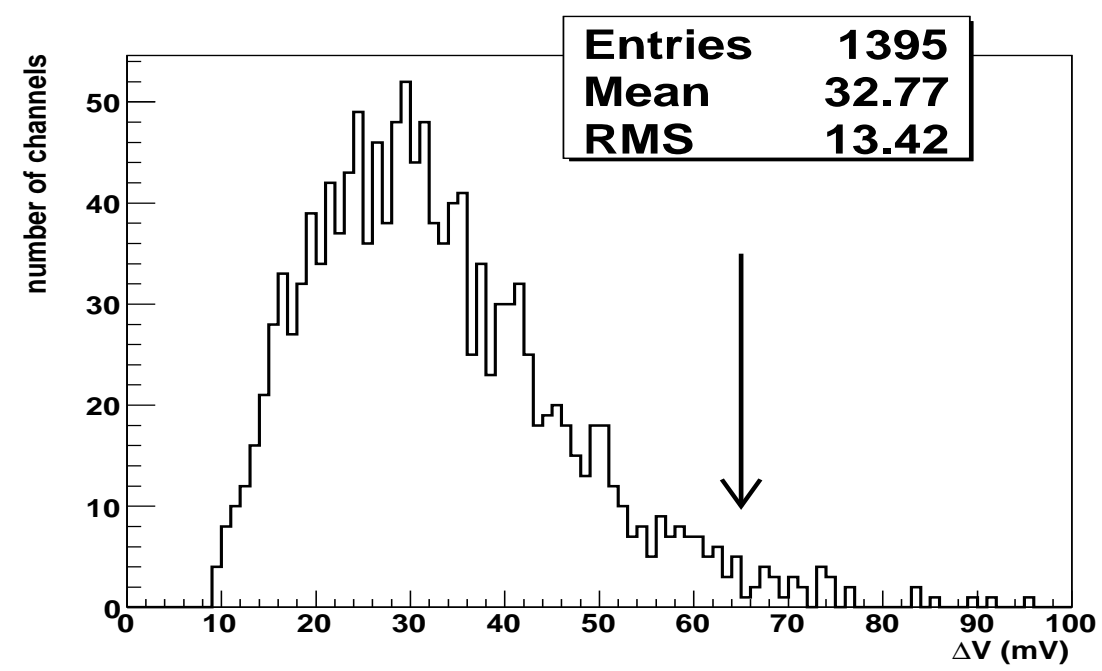

Figure 6: Distribution of $\Delta V_{\max }$, the maximum difference between measured Voltage and $V_{\text {set }}$; the channels having a value greater than $65 \mathrm{mV}$ are rejected.

In the beam tests, the APDs were biased at nominal gain 50, while, in the cosmics calibration set-up, the APDs were biased with gain 200 , to be able to select single crystal cosmics by vetoing on the surrounding ones. In all these tests the noise performance of the ECAL was very good and the average noise was, as expected, around 40 $\mathrm{MeV}$ in the single crystal [5]. This noise performance meets the design goal.

A test beam with high energy electrons was done, during summer 2006, on supermodule number 22 (SM22) in order to inter-calibrate the crystals. The test was repeated twice, with a delay of 45 days between the two tests. One of the HV boards was replaced in between the two scans because of a fault after a power cut. Figure 7 shows the distribution of the relative difference between the calibrations obtained in the two scans. This map does not show an indication of a correlation between crystal channels that belong to the same HV group (the HV channel structure is shown by the grid). Figure 8 shows the relative difference between the two calibrations averaged over each $\mathrm{HV}$ channel, versus the HV channel number. Finally, Figure 9 shows the distribution of this quantity, superimposed with a gaussian fit. The gaussian fit has $\sigma \simeq 0.1 \%$. This result shows that the long term stability and reproducibility of the HV system is well within the specifications.

\section{Integration in the ECAL detector control system}

The ECAL detector control system (DCS) [6] comprises the monitoring of the detector status, in particular various kinds of environmental parameters, as well as the detector safety system (DSS), which will generate alarms and hardwired interlocks in case of situations which could lead to damaging the detector hardware. The whole DCS software is based on the commercial SCADA package PVSS II [7]. A distributed system is built out of several applications dedicated to the DCS sub-systems. Every application is implemented as a Finite State Machine (FSM) and linked to a supervisory level, which summarizes the overall ECAL DCS status and itself incorporates a FSM. Finally, this ECAL DCS supervisor is linked to the general CMS DCS supervisory node, in order to communicate the status and alarms and to receive commands which are propagated down to the relevant sub-systems.

The HV is controlled by one of these applications, with a dedicated FSM. The PVSS application comunicates with the crate via the CAEN OPC server using the TCP/IP connection. The functionalities of this application allow part of or the whole detector to be turned on and off, the monitoring of the currents and voltages of the channels, with the display of plots of their history, and the configuration of the HV channels with various sets of voltages. The ECAL PVSS application uses a configuration database to store the values of the high voltages for various configurations and a condition database to store the monitored values of the currents and voltages and the actual HV sets used.

The ECAL Safety System in case of need can switch off the HV system either via the crate interlock located in the front panel or via the individual board interlocks. The latter solution allows the flexibility to turn off all the channels of the same supermodules at a time and will be adopted for the use in the experiment. 


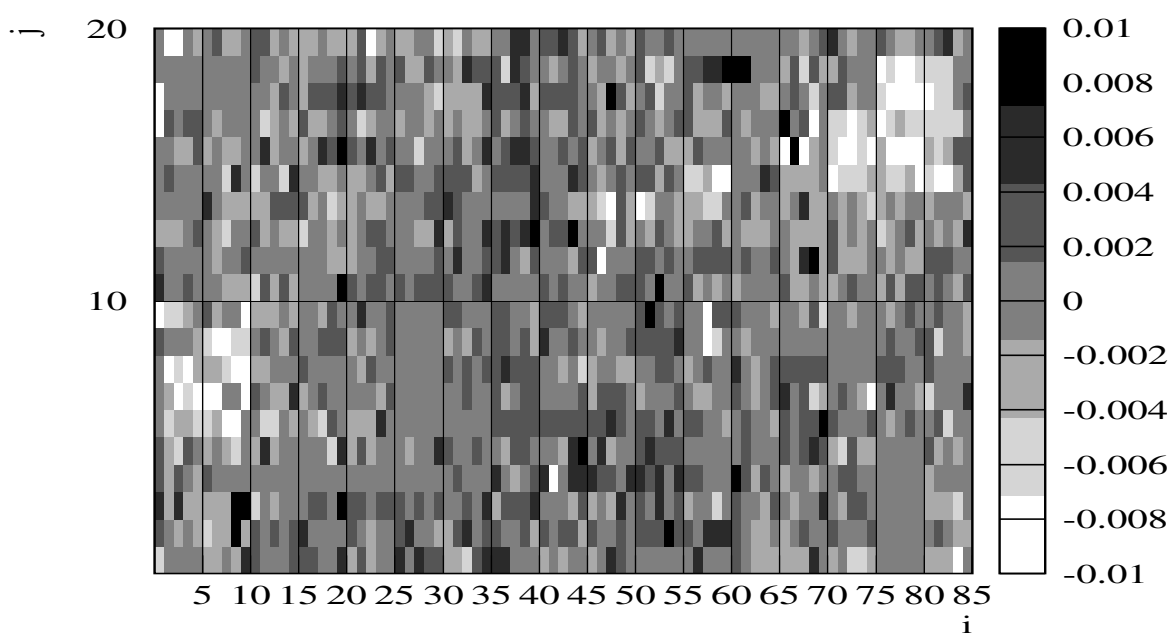

Figure 7: Relative difference between the calibrations obtained in the two scans on SM22 (2006 test beam with the final HV system with final length cables). The grid lines delimit the crystals in the same HV channel. $i$ and $j$ are the crystal indices. No evident correlation is observed between crystals in the same HV channel. Small regions of the supermodule are excluded in this analysis because they were not included in both calibration scans (156 out of 1700 crystals).

\section{Conclusions}

The High Voltage power supply system, developed for CMS ECAL, has been described in this paper. The HV system long and short term stability will allow to hold down the contribution to the energy resolution constant term at the level of $0.2 \%$. The calibration procedures and the stability tests, performed on each HV board, are reported in details. The system has been tested in the laboratory and during several ECAL test beam campaigns; the observed performances show that the HV system is within the specifications.

\section{References}

[1] CMS Collaboration, "The Compact Muon Solenoid Technical Proposal”, CERN/LHCC 94-38 CMS, LHCC/P1 (1994).

[2] CMS Collaboration, “The Electromagnetic Calorimeter Technical Design Report”, CERN/LHCC 97-33 CMS TDR 4, (1997).

[3] Z. Antunovic et al., "Radiation hard avalanche photodiodes for the CMS detector", Nucl. Instr. and Meth. A537 (2005) 379, and references therein.

[4] L.M. Barone et al., "REDACLE: A flexible database for traceability and workflow management for detector construction”, Computer Physics Communications 174 (2006) 869.

[5] CMS Ecal Electromagnetic Calorimeter Group, P. Adzic et al., "Energy resolution performance of the CMS electromagnetic calorimeter", CMS Note 2006/140.

CMS Ecal Electromagnetic Calorimeter Group, P. Adzic et al., "Energy Resolution of the Barrel of the CMS Electromagnetic Calorimeter", CMS Note 2006/148.

[6] P. Adzic et al., "The detector control system for the electromagnetic calorimeter of the CMS experiment at the LHC", CERN-CMS-CR-2005-028, presented at 10th International Conference on Accelerator and Large Experimental Physics Control Systems (ICALEPCS 2005), Geneva, Switzerland, 10-15 Oct 2005.

[7] http://www.pvss.com 


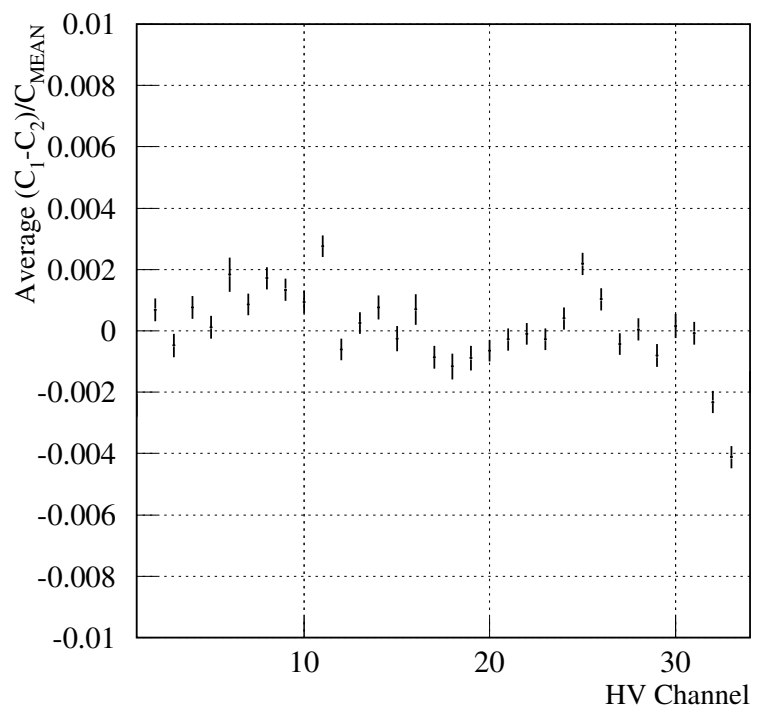

Figure 8: Relative difference between the two calibrations averaged over each HV channel, versus the HV channel number. The error bars represent the statistical error.

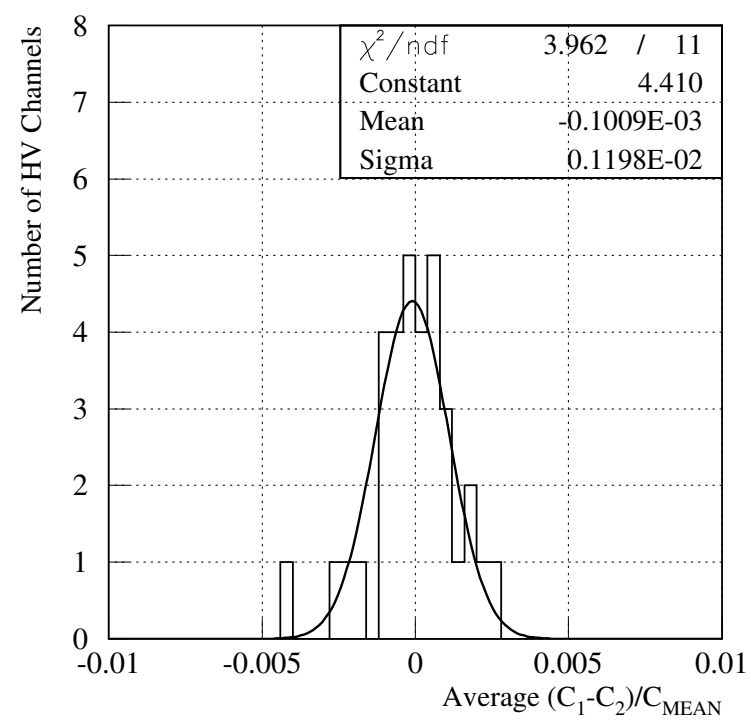

Figure 9: Distribution of the relative difference between the two calibrations averaged over each HV channel, superimposed with a gaussian fit. 